\title{
Improvement of Thermocline Structure that Affect ENSO Performance in a Coupled GCM
}

\author{
Yukiko Imada and Masahide Kimoto \\ Center for Climate System Research, The University of Tokyo, Kashiwa, Japan
}

\begin{abstract}
Many coupled atmosphere-ocean general circulation models (AOGCMs) have difficulty reproducing the El Niño/Southern Oscillation (ENSO) phenomenon. The AOGCM MIROC underestimates ENSO variability. This paper investigates the dependence of the simulated ENSO amplitude on the treatment of ocean subsurface processes.

Excessive vertical diffusivity in the subsurface ocean interferes the reproduction of a tightly stratified thermocline because the MIROC model includes numerical instability in the vertical diffusion computation and uses a large background diffusivity. The introduction of a stable numerical scheme and small background diffusivity helped improve the stratification. The improved thermocline enhanced the ENSO amplitude because the vertical heat exchange between above and below the thermocline was enhanced during the El Niño phase.
\end{abstract}

\section{Introduction}

Recently, the accuracy of coupled ocean-atmosphere general circulation models (AOGCMs) has improved dramatically because of continuous effort and the development of high-performance computers that allow highresolution modeling. However, AOGCMs still have difficulties. The variability in the El Niño/Southern Oscillation (ENSO) simulated by many AOGCMs is smaller than the observed variability. Several factors that affect the amplitude of ENSO variability have been discussed.

One of the factors that affects the ENSO amplitude is related to the equatorial easterly wind. A simulated cold tongue tends to expand to the west of the observed tongue because the easterly wind in the western tropical Pacific is overestimated (Meehl et al. 2001). As a result, the maximum ENSO amplitude simulated by a model is farther west in the equatorial Pacific than observed (Mechoso et al. 1995; Latif et al. 2001). In contrast, AOGCMs tend to underestimate the simulated trade wind in the eastern equatorial Pacific. The strength of the zonal wind stress and its anomalies affect the coupling strength (Neelin et al. 1992), and should influence El Niño variability. The model experiments by Wittenberg (2002) indicated that the ENSO shifts to a weaker amplitude, longer period, and farther east as the trade wind weakens.

Meehl et al. (2001) compared several experiments using two AOGCMs that had different model settings, such as resolution, horizontal viscosity and diffusivity, and the parameterization of the oceanic mixed layer. They showed that models with stronger zonal gradients of sea surface temperature (SST) have larger ENSO amplitudes. This tendency is related to the structure of the subsurface ocean. Models with a sharper or shallower equatorial thermocline tend to simulate a greater ENSO

Corresponding author: Yukiko Imada, Center for Climate System Research, The University of Tokyo, 5-1-5 Kashiwanoha, Kashiwa, Chiba 277-8568, Japan. E-mail: kanamaru@ccsr.utokyo.ac.jp. (C2006, the Meteorological Society of Japan. amplitude. It was also pointed out that the ocean background vertical diffusivity affects thermocline structure (Meehl et al. 2001; Wilson 2002): the lower the background diffusivity, the greater the resulting ENSO variability will be.

Although several factors influencing ENSO amplitude have been suggested, it is still not completely clear how they affect ENSO processes. We carried out experiments that examined the sensitivity of the AOGCM MIROC2.1, developed jointly by CCSR ${ }^{1}$ and NIES ${ }^{2}$ to the thermocline structure. We investigated the role of the thermocline structure in the ENSO amplitude in two experiments that differed in the stratification of the subsurface ocean.

\section{Model description and climatology}

The atmosphere part of MIROC2.1 consists of CCSR/NIES AGCM ver. 5.6, which has a spectral resolution of T42 with 20 vertical levels. The ocean component is CCSR OGCM COCO with a horizontal resolution of $1.4^{\circ}$ longitude and $0.5^{\circ}$ latitude near the equator, with 43 vertical levels. The Noh and Kim (1999) oceanic vertical mixing scheme (N-K model) is used, and no flux correction is applied. Note that the model used here is the version of MIROC preceding that used for the next Intergovernmental Panel on Climate Change (IPCC) report (Fourth Assessment Report). In this study, we analyzed 50-year integrations after a 60-year spin-up for all of the experiments.

The standard deviation of the Niño3 SST anomalies (averaged SST anomalies over the region $150^{\circ} \mathrm{W}-90^{\circ} \mathrm{W}$ and $5^{\circ} \mathrm{S}-5^{\circ} \mathrm{N}$, which serves as a proxy for the ENSO amplitude) is $0.42^{\circ} \mathrm{C}$ in MIROC2.1 compared to an observed $0.81^{\circ} \mathrm{C}$. The factors affecting the ENSO amplitude introduced in the previous section are examined in MIROC2.1. The left panels in Fig. 1 compare regression maps of SST against the Niño3 SST anomalies simulated by the model (center) with observations (top; the bottom panel is described below). The positive anomaly simulated by the model is seen not only in the eastern Pacific, but also throughout the tropical Pacific. The maximum positive simulated anomaly is near the center of the Pacific, farther west of the observed maximum. One reason for this is the stronger trade winds of the model in the western tropical Pacific (Fig. 2). In contrast, in the central and eastern tropical Pacific, the easterly wind in the model is weaker than the observed wind. These trade-wind biases weaken the ENSO variability in MIROC2.1, as well as in many other AOGCMs.

The left column of Fig. 3 shows the thermocline structure; the contour lines indicate the vertical profiles of ocean subsurface temperature along the equator. The temperature gradient near the 50-200 m depth in the equatorial region is weaker in the model (left-center) compared to the observed gradient (left-top; the other panels are mentioned later). When we define the thermocline depth as the depth of the $20^{\circ} \mathrm{C}$ isotherm at $120^{\circ} \mathrm{W}$, like Meehl et al. (2001), the thermocline in the model is about $15 \mathrm{~m}$ deeper than the observed value,

${ }^{1}$ Center for Climate System Research, The University of Tokyo ${ }^{2}$ National Institute for Environmental Studies 

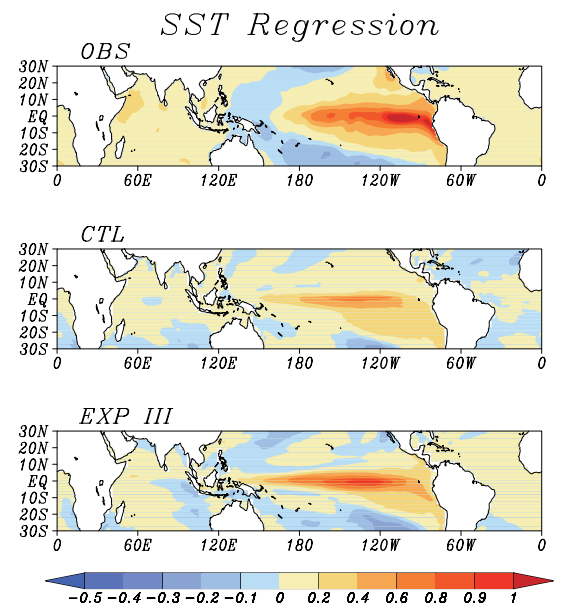

Fig. 1. Regression maps of SST against the Niño3 index $\left[{ }^{\circ} \mathrm{C}\right]$. (top: observed by Ishii et al. 2003, center: the control run, bottom: EXP. III)

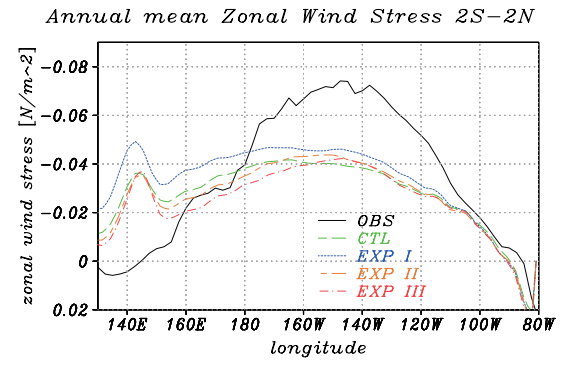

Fig. 2. Annual mean zonal wind stresses along the equator [N/ $\mathrm{m}^{2}$. (black: observed by ERA40, green: the control run, blue: EXP I, orange: EXP II, red: EXP III)

which is related to the underestimate of the ENSO amplitude variability. Consequently, MIROC2.1 has a cold SST bias in the tropical Pacific, a problem common to many AOGCMs (Neelin et al. 1992). A colder SST weakens the contrast between the waters above and below the thermocline.

Willson (2002) suggested that the parameterization of the vertical diffusivity in the oceanic mixed layer affects subsurface stratification. Figure 4 shows profiles of the vertical eddy diffusivity coefficient $\left(K_{H}\right)$. They have values exceeding $10^{3} \mathrm{~cm}^{2} / \mathrm{s}$ around the equatorial thermocline in the control run (top). In contrast, Gregg (1987) estimated the diffusivity to be on the order of 1 to $10^{2} \mathrm{~cm}^{2} / \mathrm{s}$ for the equatorial thermocline at $139^{\circ} \mathrm{W}$. The excessive diffusivity in the model disrupts a strong thermocline. The same estimation method applies to the viscosity coefficient $\left(K_{M}\right)$ (data not shown). A large viscosity at the thermocline weakens the equatorial undercurrent. In the next section, we apply appropriate methods to the vertical mixing computation to remove large mixing around the thermocline.

\section{Experiments}

The extreme diffusivity and viscosity in our model are attributable to two factors. First, numerical instability produces a diffuse thermocline when an implicit method is used for the nonlinear terms in a vertical mixing scheme. We apply the time-filtering scheme of Kalnay and Kanamitsu (1988), in which the mixing coefficient value is averaged with the value from the previous step before integration. This method reduces the numerical instability in the vertical mixing calculation. We call this experiment EXP I.

Second, a model cannot maintain a state absent of diffusion or viscosity at the oceanic boundary layer because of the large minimum settings of the mixing $\mathrm{CO}^{-}$ efficient. The N-K model used in MIROC2.1 solves an equation for the evolution of the turbulence kinetic energy (TKE), and calculates the mixing coefficient as a function of the vertical gradient of the mean buoyancy, TKE, and a roughness length scale. As the Richardson number of the turbulence increases, the mixing coefficients decreases and ultimately reaches the background mixing setting. Therefore, lower background values allow the N-K model to calculate more conditions under which diffusion and viscosity are absent. We reduced the background values of the $K_{H}$ and $K_{M}$ within the limits $0.01 \mathrm{~cm}^{2} / \mathrm{s}$ and $0.2 \mathrm{~cm}^{2} / \mathrm{s}$, respectively, based on the estimation by Gregg (1987) for near the ocean surface (from the surface to a depth of $500 \mathrm{~m}$ ), where a tightly stratified thermocline exists. We call this experiment EXP II. In the following section, we compare the subsurface stratification and ENSO variability of these modified runs with the control run.

\section{Results}

The vertical diffusivity simulated in EXP I is shown in Fig. 4 (center panel). After removing the numerical instability, the excessive diffusivity disappeared, except for a well mixed surface mixing layer. However, the region around the thermocline in the eastern equatorial Pacific had a diffusivity equal to that of the well mixed warm-pool regions above the thermocline. Furthermore, the diffusivity values corresponded to the background value below the tropical thermocline and around the thermocline in the western tropical Pacific in EXP I. This means that this model could not calculate a diffusivity value less than the background value, even if a greater condition without diffusion were possible in the subsurface ocean. The viscosity had characteristics similar to the diffusivity.

To deal with the saturation of these values, we set the background vertical diffusivity and viscosity in EXP II to sufficiently smaller values. The $K_{H}$ distribution in EXP II is shown in Fig. 4 (bottom panel). The modification was effective along the equatorial thermocline in the western Pacific. This made the thermocline region more stable than the surrounding layers, sharpening the equatorial thermocline. The equatorial undercurrent along the equatorial thermocline was also intensified because the instability produced by the background viscosity was reduced (data not shown).

A more serious cold bias of the equatorial SST was observed in EXP II than in the control run because a sharper thermocline induces the ascent of cold water. To reduce the equatorial SST biases, in EXP III, we introduced parameterizations such as a nonlocal planetary boundary layer (Troen and Mahrt 1986; Holtslag 1993) and cloud top entrainment instability (Deardorff 1980; Randall 1980), which improved the dissipation of cloud water and dried the top of the atmospheric boundary layer. The original model had a positive bias for stratus cloud in the tropical Pacific. By considering these processes, the SST increased because of the reduction in tropical stratus cloud, although it was still overestimated (details not given). This experiment called EXP III is our final result. The contour lines in the leftbottom panel of Fig. 3 show the seawater temperature for EXP III. The thermocline was sharper in EXP III compared to the control. The tropical zonal SST gradient recovered because of the sharpened thermocline. The overestimate of the trade winds in the western tropical Pacific was ameliorated (Fig. 2) owing to the improved SST distribution. However, all the runs still underestimated the easterly wind in the central and eastern tropical Pacific, suggesting another reason for 

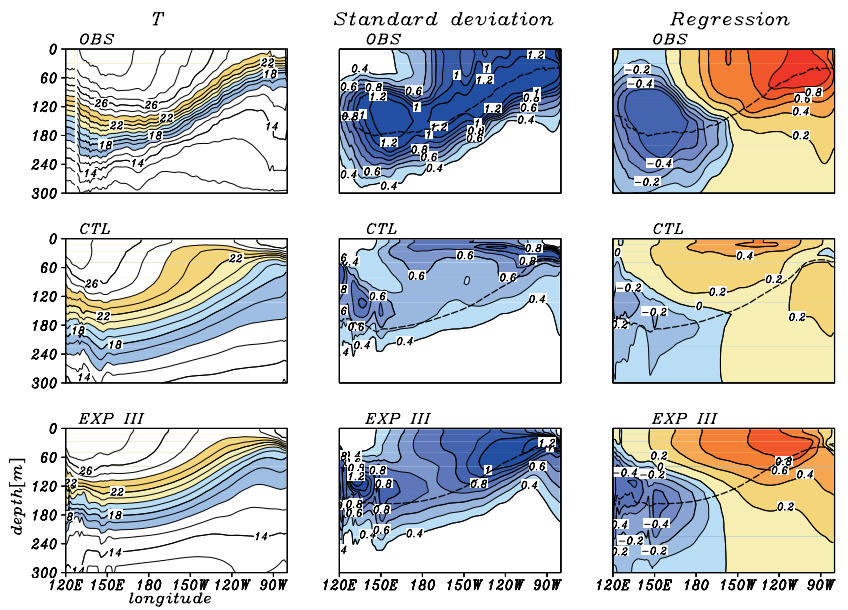

Fig. 3. Annual mean seawater temperatures $(T)$ (the left column), interannual standard deviations of $T$ (the center column), and regression of $T$ against the Niño3 index (the right column) in the equatorial subsurface Pacific ocean (unit: ${ }^{\circ} \mathrm{C}$ ). A dash line indicates the $20{ }^{\circ} \mathrm{C}$ isotherm. (top: observed by Ishii et al. 2003, center: the control run, bottom: EXP III).

the calculated weak trade winds. The wind bias remains one of the problems to be improved in MIROC2.1, as well as in many other AOGCMs.

The changes in the thermocline structure in EXP III affected the interannual fluctuations in the subsurface ocean. The standard deviation of the equatorial subsurface temperature anomalies are shown in the center column of Fig. 3. In the control run, when the equatorial thermocline was diffuse, the fluctuations in the equatorial subsurface were smaller than the observed values. Most of the variability in the control run occured near the surface of the central Pacific. In EXP III, the sharper thermocline resulted in greater variability than in the control, especially when it resembled the observed thermocline. These fluctuations in the subsurface ocean are tightly connected to the ENSO variability. The right column in Fig. 3 shows the regression maps of sea temperature against Niño3 SST anomalies. Both positive and negative anomalies existed on the thermocline in the eastern and western equatorial Pacific, where the interannual fluctuation in the observed temperature became large (right-top), and in EXP III (right-bottom). In the control run (right-center), the positive anomalies were trapped near the surface above the thermocline in the central Pacific because the region along the thermocline did not fluctuate owing to the diffuse temperature gradient. The standard deviation of the Niño3 SST anomalies increased to $0.46,0.56$, and $0.60^{\circ} \mathrm{C}$ in EXP I to EXP III, respectively.

To investigate how the modifications of the tropical subsurface structure affect the ENSO amplitude, we compared the budgets of the upper ocean temperature among the runs using the following equation:

$$
\begin{aligned}
\int_{z=50 m}^{z=0 m} \frac{\partial T^{\prime}}{\partial t} d z= & -\int_{z=50 m}^{z=0 m}\left[U \frac{\partial T^{\prime}}{\partial x}+u^{\prime} \frac{\partial\left(T+T^{\prime}\right)}{\partial x}\right] d z \\
& -\int_{z=50 m}^{z=0 m}\left[V \frac{\partial T^{\prime}}{\partial y}+v^{\prime} \frac{\partial\left(T+T^{\prime}\right)}{\partial y}\right] d z \\
& -\int_{z=50 m}^{z=0 m}\left[W \frac{\partial T^{\prime}}{\partial z}+w^{\prime} \frac{\partial\left(T+T^{\prime}\right)}{\partial z}\right] d z+Q^{\prime} \\
& -\left.K_{H} \frac{\partial T^{\prime}}{\partial z}\right|_{z=50 m},
\end{aligned}
$$

where $T, U, V$, and $W$ are the monthly mean sea temperature, and zonal, meridional, and vertical velocities,
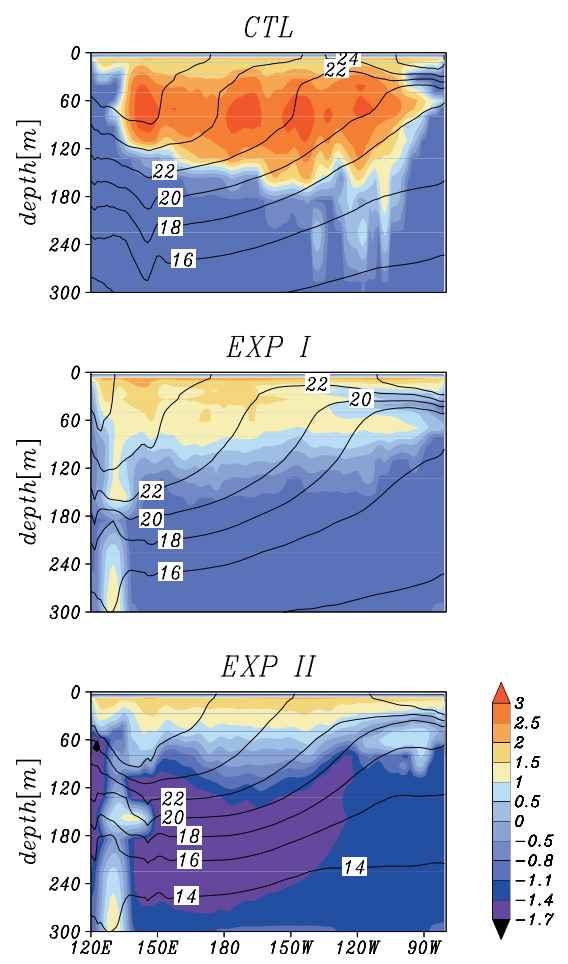

Fig. 4. Annual mean vertical diffusivity coefficients (shade; unit: $\mathrm{cm}^{2} / \mathrm{s}, \log$ scale) and isotherms (contour; unit: ${ }^{\circ} \mathrm{C}$ ) in the equatorial subsurface Pacific ocean. (top: the control run, center: EXP I, bottom: EXP II)

respectively (following lizuka et al. 2003). The prime symbols denote each perturbation. $Q^{\prime}$ is anomalous heating resulting from radiation, latent heat, and sensible heat. We compared these terms with regression maps against the Niño3 SST anomalies from -2- to +2year lags on the equator. The correlations of each regression map for the right-hand-side terms against the tendency of the SST anomalies (left-hand side) are shown in Fig. 5. These indices represent the efficiency of each budget term affecting SST variability during the ENSO cycle. The result showed that the predominant contributor to SST changes was the mean vertical advection, the upwelling term that advects temperature anomalies, $-W\left(\partial T^{\prime} / \partial z\right)$, which resulted from the mean advection of anomalous temperature by the mean upwelling in EXP III. The anomalous zonal current, $-u^{\prime} \partial\left(T+T^{\prime}\right) / \partial x$, worked positively in both runs. Kang et al. (2001) also noted similar structures for the dominant terms. The contribution of anomalous upwelling, $-w^{\prime} \partial T / \partial z$, in EXP III and the negative contribution of diffusivity in both runs was also relatively large. We make no reference to these two budgets because they resulted from the variance in the far eastern Pacific (data not shown), where the accuracy of AOGCMs with similar resolution to MIROC2.1 is poor. The contributions of meridional advection terms were relatively small in both runs because these budgets were calculated right at the equator, where the mean meridional current essentially vanishes. Conversely, the mean zonal advection of the temperature anomalies, $-U\left(\partial T^{\prime} / \partial x\right)$, contributed predominantly in the control run. These results indicate that heat exchange across the thermocline via equatorial upwelling was activated with a sharpened thermocline in EXP III. In the control run, the upwelling was not effective because the thermocline was diffuse. By way of compensation, the SST changes in an ENSO cycle mainly owe their existence to zonal advection near the ocean surface, as mentioned in Neelin's SST mode (Neelin 1991). ${ }^{3}$ 


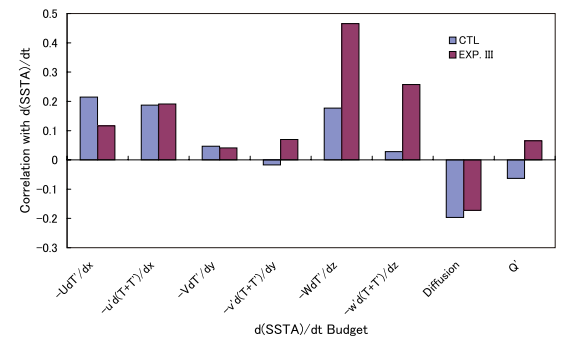

Fig. 5. Correlation coefficients of each budget of SST anomalies (integrated upper $50 \mathrm{~m}$ ) against SST tendencies (calculated by the regression against Niño3 SST anomalies from -2 to +2 years lag). CTL (blue column) and EXP III (dark red column).

\section{Discussion and conclusion}

We examined the factors affecting the amplitude of the simulated ENSO variability, and performed experiments to examine the sensitivity of the thermocline structure using an AOGCM. We then sought to elucidate a mechanism that would explain how ENSO changes in a model.

The vertical diffusivity and viscosity in the tropical subsurface ocean simulated by the AOGCM MIROC2.1 were excessive because the numerical instability in the vertical mixing computation was nonlinearly dependent on the stratification, and large background mixing occurred. Numerical instability could be removed using Kalnay and Kanamitsu's time filter. On reducing the background diffusivity and viscosity in the ocean subsurface, the region along the thermocline remained absent of diffusion and viscosity. If a field is restricted to exclude diffusion, the contrast between the well mixed upper warm layer and lower cold layer is not sustained. The eastward equatorial undercurrent, which maintains its speed in a region sufficiently devoid of viscosity, also played a role in sustaining the upper-lower contrast by offsetting the surface westward current. As a result, the model was able to retain a sharper thermocline. We also examined other factors, such as the horizontal diffusivity, and found that the modifications discussed here predominated. Recently, Noh et al. (2002) stated that the empirical constant in an ocean vertical mixing scheme, which parameterizes the effects of stratification, depends on the vertical resolution. The variation in the Prandtl number (fixed in MIROC2.1), along with stratification, is also one of the factors used to simulate the tropical thermocline structure (Noh et al. 2005). A later version of this model included this parameter, and it had a minimal effect on the problem we focused on here. However, it is important to investigate other effects of these modifications in the new version model.

With a sharp thermocline, the region of positive anomalies for the regression temperature against ENSO variability was located along the thermocline, while it was confined to near the ocean surface in the central Pacific with a diffuse thermocline. In the former case, the ENSO amplitude was intensified. A budget analysis showed that with a diffuse thermocline, surface horizontal westward advection played a role in driving the El Niño phase. This mechanism is noted in Neelin's SST model (Neelin 1991). In contrast, with a sharp thermocline, vertical advection played a more dominant role. Changes in thermocline structure affected subsurface advection, which influenced the ENSO variability. The

${ }^{3}$ The contribution of anomalous heat fluxes is insignificant in Fig. 5. This result is consistent because anomalous heat fluxes generally act to dampen SST anomalies (out of phase by $180^{\circ}$ ), i.e., it leads a tendency of SST anomalies by $90^{\circ}$. amount of background mixing largely contributed to the change in the ENSO amplitude. We suggest that the background mixing in stable regions must be defined more precisely. Furthermore, a precise estimation of wind stress will be important for the recovery of the ENSO amplitude. These issues remain for future work.

\section{Acknowledgments}

We gratefully thank Prof. M. Watanabe for giving us insightful suggestions. This work was supported by the research revolution 2002 (RR2002) program of the Ministry of Education, Culture, Sports, Science and Technology, and the Core Research foe Evolutional Science and Technology of the Japan Science. The SR8000 of Supercomputing Division, Information Technology Center, the University of Tokyo was used for the model experiments.

\section{References}

Deardorff, J. W., 1980: Cloud-top entrainment instability. J. Atmos. Sci., 37, 131-147.

Holstlag, A. A. M., and B. A. Boville, 1993: Local versus non-local boundary-layer diffusion in a global climate model. J. Clim. 6, 1825-1842.

Iizuka, S., K. Orito, and T. Matsuura, 2003: Influence of cumulus convection schemes on the ENSO-like phenomena simulated in a CGCM. J. Meteor. Soc. Japan, 81, 805-827.

Ishii, M., M. Kimoto, and M. Kachi, 2003: Historical ocean subsurface temperature analysis with error estimates. Mon. Wea. Rev., 131, 51-73.

Kalnay, E., and M. Kanamitsu, 1988: Time schemes for strongly nonlinear damping equations. Mon. Wea. Rev., 116, 19451958.

Kang, I. S., S. I. An, and F. F. Jim, 2001: A systematic approximation of the SST anomaly equation for ENSO. J. Meteor. Soc. Japan, $79,1-10$.

Latif, M., K. Sperber, and co-authors, 2001: ENSIP: the El Niño simulation intercomparison project. Clim. Dyn., 18, 255-276.

Mechoso, C. R., A. W. Robertson, N. Barth, and co-authors, 1995: The seasonal cycle over the tropical Pacific in coupled oceanatmosphere general circulation models. Mon. Wea. Rev., 123, 2825-2838.

Meehl, G. A., P. R. Gent, and co-authors, 2001: Factors that affect the amplitude of El Niño in global coupled climate models. Clim. Dyn., 17, 515-526.

Neelin, J. D., 1991: The slow surface temperature mode and the fastwave limit: analytic theory for tropical interannual oscillations and experiments in an hybrid coupled model. J. Atmos. Sci., 48, 584-606.

Neelin, J. D., M. Latif, and co-authors, 1992: Tropical air-sea interaction in general circulation models. Clim. Dyn., 7, 73-104.

Noh, Y., and H. J. Kim, 1999: Simulations of temperature and turbulence structure of the oceanic boundary layer with the improved near-surface process. J. Geophys. Res., 104, 1562115634.

Noh, Y., C. J. Jang, T. Yamagata, P. C. Chu, and C. H. Kim, 2002: Simulation of more realistic upper-ocean processes from an OGCM with a new ocean mixed layer model. J. Phys. Oceanogr., 32, 1284-1307.

Noh, Y., and Y. J. Kang, 2005: Effect of the Prandtl number in the parameterization of vertical mixing in an OGCM of the tropical Pacific. Geophys. Res. Lett., 32, doi: 10.1029/ 2005GL024540.

Randall, D. A., 1980: Conditional instability of the first kind upsidedown. J. Atmos. Sci., 37, 125-130.

Troen, I., and L. Mahrt, 1986: A simple model of the atmospheric boundary layer: Sensitivity to surface evaporation. Bound.Layer Meteor., 37, 129-148.

Willson, S. G., 2002: Evaluation of various vertical mixing parameterizations in a tropical Pacific Ocean GCM. Ocean Modell., 4, 291-311.

Wittenberg, A. T., 2002; ENSO response to altered climates. Ph. D. thesis, Prinston University, $475 \mathrm{pp}$.

Manuscript received 30 August 2006, accepted 27 October 2006

SOLA: http://www.jstage.jst.go.jp/browse/sola/ 